\title{
Qualidade microbiológica de fórmulas infantis administradas em hospital público do município de Campinas, São Paulo
}

\author{
Andressa Reginato ${ }^{1}$, Fabíola de Lima Pena ${ }^{2}$, Fabiana K.S Trento ${ }^{3}$, Luciane Cristina Rosim \\ Sundfeld Giordano ${ }^{4}$, Harumi Kinchoku ${ }^{5}$ e Adriane Elisabete Costa Antunes ${ }^{6}$
}

A qualidade microbiológica em lactários é um item que merece toda a atenção e deve ser mantida de forma rigorosa. Considerando a população alvo desses serviços e o ambiente em que estão inseridos, sabe-se que são locais de alto risco para contaminações microbiológicas e as consequências destas podem ser fatais. Assim, o objetivo deste estudo foi verificar a qualidade microbiológica de fórmulas infantis utilizadas para alimentação de crianças internadas na unidade de pediatria de um hospital público do município de Campinas - SP. Foram realizadas análises microbiológicas de bactérias aeróbias mesófilas totais, coliformes totais e termotolerantes e Estafilococos coagulase positiva das fórmulas infantis, comparando-se resultados com os valores especificados na Resolução da Diretoria Colegiada n⿳o 12, de 02 de janeiro de 2001 (RDC 12/2001), da Agência de Vigilância Sanitária. Das 26 amostras de fórmulas infantis, 88,5\% atenderam aos parâmetros para oferecimento para menores de 1 ano de vida, e 92,3\% atenderam para as exigências para pacientes com mais de 1 ano de vida, segundo a RDC 12/2001. Porém, quando observados paralelamente adequação a parâmetros desta legislação e a recomendação da Food and Drug Administration (2006) para microrganismos aeróbios mesófilos, apenas $46,2 \%$ apresentavam contagens dos microrganismos avaliados dentro dos limites tolerados.

Palavras-chave: fórmulas infantis, microbiologia, segurança dos alimentos.

\section{Microbiological quality of infant formula administered in a public hospital of Campinas - SP}

The microbiological quality at lactaries is an item that deserves attention and should be keeping strictly. There are high microbiological risks of contamination at the hospital and the consequence of this can be fatal considering the target population of those services. The objective of this study was to determine the microbiological quality of infant formulas used for feeding infants hospitalized at a pediatric unit of a hospital in the city of Campinas SP. The infant formulas were evaluated for aerobic mesophilic bacteria, coliforms at $30-35^{\circ} \mathrm{C}$ and $45^{\circ} \mathrm{C}$, and

\footnotetext{
${ }^{1}$ Faculdade de Ciências Aplicadas, Universidade Estadual de Campinas.

${ }^{2}$ Faculdade de Ciências Aplicadas, Universidade Estadual de Campinas.

${ }^{3}$ Centro de Pesquisa e Desenvolvimento de Laticínios, Instituto de Tecnologia de Alimentos, Campinas.

${ }^{4}$ Hospital de Clínicas (HC), Universidade Estadual de Campinas.

${ }^{5}$ Hospital de Clínicas (HC), Universidade Estadual de Campinas.

${ }^{6}$ Faculdade de Ciências Aplicadas, Universidade Estadual de Campinas. Correspondência: Rua Pedro Zaccaria no 1.300, Jardim São Paulo, Limeira, SP, Brasil. CEP: 13484-350.Telefone: (19)3701-6650.E-mail: adriane.antunes@fca.unicamp.br
} 
Staphylococcus coagulase positive and the results compared to Resolution № 12 of 02 January 2001, of the Health Surveillance Agency from Brazil. Twenty-six samples of infant formula $(88.5 \%)$ met the criteria to be offered to younger than 1 year old babies and $92.3 \%$ met the criteria to be offered to childs older than 1 year, according to RDC 12/2001 observing coagulase-positive staphylococci and coliforms. However, when taking in consideration also the parameters from FDA (2006) for mesophilic aerobic counts only $46.2 \%$ of the samples were within the tolerance limits regarding the microorganisms evaluated.

Key-words: infant formulas, microbiology, food safety.

\section{INTRODUÇÃO}

O leite materno é o primeiro, e possivelmente o mais completo dos alimentos ingeridos pelo homem e demais mamíferos, visto que apresenta um adequado equilíbrio de macro e micronutrientes, satisfazendo todas as necessidades nutricionais dos recém-nascidos nos primeiros meses de vida ${ }^{[1]}$.

O aleitamento materno, entretanto, pode ser impedido por situações temporárias ou permanentes, tais como em algumas doenças infecciosas (varicela, herpes com lesões mamárias, tuberculose não tratada, infecção pelo vírus da imunodeficiência humana), pelo uso de medicamentos passíveis de liberação pelo epitélio alveolar mamário e nocivos ao bebê. Outros fatores são devido à ocorrência de doenças metabólicas raras do bebê como a fenilcetonúria e a galactosemia [2].

Diante de tais situações, a solução é o oferecimento de fórmulas à base de leite de vaca ou produtos à base de soja para a alimentação do recémnascido [3].

Por definição, fórmulas infantis são produtos na forma pó ou líquida, utilizados sob prescrição do médico ou nutricionista, para satisfazer as necessidades nutricionais de lactentes sadios ou enfermos e atuar como complemento na alimentação de crianças na primeira infância. Estes produtos podem ter como base o leite de vaca ou de outros animais ou ainda a mistura deste e/ou de outros ingredientes que sejam comprovados como adequados para alimentação destes grupos etários [4].

Ressalta-se que, embora as fórmulas infantis ainda não tenham conseguido reproduzir as propriedades imunológicas e de digestibilidade do leite materno até o momento, elas ainda atendem as necessidades nutricionais estimadas, segundo Codex
Alimentarius - FAO/WHO [5], quando utilizadas como fonte única de nutrientes durante os primeiros seis meses de idade [6]. Após o sexto mês de vida, é necessária a inclusão de outros alimentos para se atingir as recomendações dietéticas do bebê. Quando existe a possibilidade de aleitamento materno, recomenda-se que este seja mantido até os 2 anos de idade ou mais, em conjunto com a alimentação complementar iniciada após os primeiros 6 meses de vida [7].

Além de desempenhar o papel nutritivo, os alimentos destinados ao público infantil devem ser seguros para o consumo. Conforme descrito por Silva e Cardoso (2011), a segurança dos alimentos é definida pela FAO/WHO como a garantia de que o consumidor não sofrerá danos após consumo de um alimento. Frente a isto, o alimento deve ser caracterizado como inócuo à saúde, sendo livre de contaminantes químicos, físicos e microbiológicos ${ }^{[8]}$.

Ao ponderar sobre a segurança microbiológica das fórmulas infantis é importante salientar que o leite de vaca, componente base da maior parte das fórmulas infantis, possui características que permitem que seja um excelente meio de cultura para a multiplicação de microrganismos, principalmente devido à presença de gorduras emulsificadas, concentrações fisiológicas de proteínas, conteúdo de sais e açúcares, além de $\mathrm{pH}$ em torno de 6,8 []. Esta condição reafirma a importância nos cuidados do preparo destas formulações.

Considerando a seriedade e importância de manter a segurança destes alimentos, primeiramente, as empresas produtoras de fórmulas lácteas e alimentos para público infantil precisam atender a exigentes critérios de higiene e segurança. As principais legislações brasileiras que abordam e regulamentam as fórmulas infantis são a Portaria no 977, de 5 de dezembro de 1998 [9] e as Resoluções da Diretoria Colegiada - RDC 43 e 44 de 19 de setembro de 2011, 
publicadas pela Secretaria de Vigilância Sanitária do Ministério da Saúde [4].

No ambiente hospitalar - principal local de manipulação destes produtos - as fórmulas infantis são geralmente preparadas em área específica denominada Lactário: unidade destinada à higienização, ao preparo, armazenamento e à distribuição das mamadeiras com fórmulas infantis lácteas e não lácteas e/ou seus substitutos para a alimentação de lactentes internados na enfermaria de pediatria ${ }^{[10]}$.

A etapa de preparo nesta área pode representar um risco do ponto de vista higiênicosanitário pela introdução de patógenos nas fórmulas infantis originalmente seguras para consumo. Sendo assim, existem também nesta fase da produção destes alimentos, rigorosos protocolos de higiene que devem ser seguidos, relacionados à higiene dos manipuladores e padronização de procedimentos de boas práticas de preparo das mamadeiras com fórmulas infantis e seus substitutos, ao ambiente, higienização de utensílios. Tais medidas devem abranger cuidados higiênicosanitários compatíveis com a prevenção de doenças infecciosas [11]. Os principais problemas microbiológicos relacionados com leite em pó e produtos derivados, como as fórmulas infantis em pó, ocorrem devido à contaminação acidental durante, ou após a reconstituição em meio líquido [12].

Rossi (2007) comenta em seu estudo que infecções ao longo do primeiro ano de vida são as principais causas da elevação do índice de morbimortalidade entre os lactentes [13]. Diante disso destaca-se que a presença de determinadas espécies de microrganismos pode interferir no quadro clínico de bebês e crianças hospitalizadas. Por exemplo, o $S$. aureus enterotoxigênico produz toxinas resistentes à pasteurização, podendo acarretar sérios problemas como diarreia, náusea, vômito, febre, calafrio e prostração [14].

Pelo presente exposto, este trabalho teve por objetivo verificar a qualidade microbiológica de fórmulas infantis manipuladas em área de Lactário e distribuídas na unidade de internação da pediatria de um hospital público do município de Campinas - SP.

Os valores obtidos nas análises das fórmulas infantis foram comparados com a Resolução da Diretoria Colegiada (RDC) no 12, de 02 de Janeiro de 2001, que regulamenta os padrões microbiológicos deste alimento no país [15], e com o Compliance Program Guidance Manual da Food and Drug Administration [16] que também dispõe sobre parâmetros microbiológicos do alimento.

\section{METODOLOGIA}

Esta pesquisa foi conduzida na área de Lactário de um hospital público no município de Campinas, São Paulo, Brasil, onde são preparadas as fórmulas infantis e sucos de frutas in natura, armazenadas sob refrigeração e distribuídas para lactentes internados na unidade de internação da pediatria.

A preparação das fórmulas no lactário obedece a um fluxo padrão do serviço, no qual as fórmulas infantis em pó são preparadas com uso de água potável, com homogeneização através de liquidificador previamente higienizado com água potável e detergente sanitizante. Após a homogeneização o conteúdo é transferido para jarras plásticas com tampa até o momento de porcionamento em mamadeiras higienizadas e esterilizadas em autoclave. As mamadeiras porcionadas são rotuladas e armazenadas sob refrigeração $\left(5,3 \pm 1^{\circ} \mathrm{C}\right)$ em "pass-through" refrigerado até $\mathrm{o}$ momento de distribuição pelas copeiras, quando então é realizado o aquecimento em banho-maria.

\section{Coleta de amostras}

Para a coleta das amostras a serem analisadas foram selecionadas sete marcas comerciais de fórmulas infantis diferentes. Algumas marcas foram coletadas e analisadas por até cinco vezes distintas, resultando em um total de 26 amostras de fórmulas infantis.

As amostras deste estudo foram obtidas a partir de amostras coletadas rotineiramente das preparações que eram separadas pelos funcionários do Lactário e armazenadas sob refrigeração por um período de 72 horas para possível realização de análise laboratorial e controle do próprio Lactário, quando necessário. Para esta pesquisa as amostras coletadas deviam estar armazenadas em um período máximo de 24 horas. Aproximadamente $60 \mathrm{~mL}$ de cada fórmula foram transferidas para tubos estéreis, do tipo Falcon, identificados e armazenados em bolsa térmica até o transporte para o laboratório de Higiene e Microbiologia dos Alimentos da Faculdade de Ciências Aplicadas da UNICAMP. 
As análises microbiológicas realizadas consistiram em contagem dos microrganismos aeróbios mesófilos totais, coliformes totais e termotolerantes (também denominados respectivamente coliformes a $30-35^{\circ}$ e coliformes a $\left.45^{\circ} \mathrm{C}\right)$ e teste de Estafilococos coagulase positiva, segundo as metodologias descritas por SILVA et al. (2010) [17], conforme sumarizado no Quadro 1.

Quadro1. Técnicas utilizadas nas análises microbiológicas.

\begin{tabular}{|c|c|c|c|c|}
\hline Microrganismos & $\begin{array}{c}\text { Meios de } \\
\text { cultura/Prova } \\
\text { bioquímica }\end{array}$ & Técnicas & Incubação & $\begin{array}{l}\text { Expressão do } \\
\text { resultado }\end{array}$ \\
\hline $\begin{array}{c}\text { Aeróbios mesófilos } \\
\text { totais }\end{array}$ & Plate Count Agar (PCA) & Pourplate & $35 \pm 1^{\circ} \mathrm{C} / 48 \pm 2 \mathrm{~h}$ & $\mathrm{UFC} / \mathrm{mL}$ \\
\hline Coliformes a $30-35^{\circ} \mathrm{C}$ & $\begin{array}{l}\text { Caldo Lauril Sulfato } \\
\text { Triptose (LST) } \\
\text { Bile Verde Brilhante } \\
\text { (VB) }\end{array}$ & $\begin{array}{c}\text { Fermentação em tubos } \\
\text { múltiplos }\end{array}$ & $\begin{array}{c}35,5 \pm 0,5^{\circ} \mathrm{C} / 48 \pm 2 \mathrm{~h} \\
35^{\circ} \mathrm{C} \pm 0,5^{\circ} \mathrm{C} / 24-48 \mathrm{~h} \pm 2 \mathrm{~h}\end{array}$ & $\mathrm{NMP} / \mathrm{mL}$ \\
\hline Coliformes a $45^{\circ} \mathrm{C}$ & $\begin{array}{l}\text { Caldo Lauril Sulfato } \\
\text { Triptose (LST) } \\
\text { Caldo EC }\end{array}$ & $\begin{array}{l}\text { Fermentação em tubos } \\
\text { múltiplos }\end{array}$ & $\begin{array}{c}35,5 \pm 0,5^{\circ} \mathrm{C} / 48 \pm 2 \mathrm{~h} \\
45,5^{\circ} \pm 0,2^{\circ} \mathrm{C} / 24 \mathrm{~h} \pm 2 \mathrm{~h} \\
\text { (banho-maria) }\end{array}$ & $\mathrm{NMP} / \mathrm{mL}$ \\
\hline $\begin{array}{c}\text { Estafilococos coagulase } \\
\text { positiva }\end{array}$ & $\begin{array}{c}\text { Agar Baird-Parker (BP) } \\
\text { Brain Heart Infusion } \\
\text { (BHI) } \\
\text { Plasma de coelho } \\
\text { EDTA } \\
\text { Catalase }\end{array}$ & $\begin{array}{c}\text { Spread plate } \\
\text { Crescimento em tubo } \\
\text { Teste de coagulação } \\
\text { Prova de aglutinação }\end{array}$ & $\begin{array}{c}35 \pm 0,2^{\circ} \mathrm{C} / 48 \pm 2 \mathrm{~h} . \\
35^{\circ} \pm 1^{\circ} \mathrm{C} / 24 \mathrm{~h} \\
35^{\circ} \pm 1^{\circ} \mathrm{C} / 6 \mathrm{~h} \\
25^{\circ} \mathrm{C} \text { (ambiente) }\end{array}$ & $\mathrm{UFC} / \mathrm{mL}$ \\
\hline
\end{tabular}

\section{RESULTADOS E DISCUSSÃO}

Os resultados das análises microbiológicas realizadas nesta pesquisa estão expressos na Tabela 1. 
Tabela 1. Resultados das análises microbiológicas das amostras de fórmulas infantis coletadas em lactário hospitalar no município de Campinas - SP e situação de adequação quanto à legislação.

\begin{tabular}{|c|c|c|c|c|c|c|c|c|c|}
\hline \multirow{2}{*}{ Amostra } & \multirow{2}{*}{$\begin{array}{l}\text { Aeróbios } \\
\text { mesófilos } \\
\text { (UFC/mL) }\end{array}$} & \multirow[t]{2}{*}{$\begin{array}{l}\text { Adequação } \\
\text { FDA (2006) }\end{array}$} & \multirow{2}{*}{$\begin{array}{l}\text { Coliformes } \\
\text { a } 30-35^{\circ} \\
(\mathrm{NMP} / \mathrm{mL})\end{array}$} & \multicolumn{2}{|c|}{$\begin{array}{l}\text { Adequação RDC } 12 \\
\text { (Brasil, 2001) }\end{array}$} & \multirow{2}{*}{$\begin{array}{c}\text { Coliformes } \\
\text { a } 45^{\circ} \\
(\mathrm{NMP} / \mathrm{mL})\end{array}$} & \multicolumn{2}{|c|}{$\begin{array}{c}\text { Adequação RDC } 12 \\
\text { (Brasil, 2001) }\end{array}$} & \multirow{2}{*}{$\begin{array}{c}\text { S. Aureus } \\
\text { Coagulase } \\
\text { positiva } \\
\text { (UFC } / \mathrm{mL} \text { ) }\end{array}$} \\
\hline & & & & $\begin{array}{l}\text { Até } 1 \text { ano } \\
\text { de idade }\end{array}$ & $\begin{array}{c}\text { Acima de } 1 \\
\text { ano } \\
\end{array}$ & & $\begin{array}{l}\text { Até } 1 \text { ano } \\
\text { de idade }\end{array}$ & $\begin{array}{c}\text { Acima de1 } \\
\text { ano }\end{array}$ & \\
\hline 1.0 & 1 & $\operatorname{sim}$ & $<3,0$ & $\operatorname{sim}$ & $\operatorname{sim}$ & $<3,0$ & $\operatorname{sim}$ & $\operatorname{sim}$ & $<10$ \\
\hline 1.1 & $<10$ & $\operatorname{sim}$ & $<3,0$ & $\operatorname{sim}$ & $\operatorname{sim}$ & $<3,0$ & $\operatorname{sim}$ & $\operatorname{sim}$ & $<10$ \\
\hline 1.2 & $2,5 \times 10^{4}$ & não & $<3,0$ & $\operatorname{sim}$ & $\operatorname{sim}$ & $<3,0$ & $\operatorname{sim}$ & $\operatorname{sim}$ & $<10$ \\
\hline 1.3 & $<10$ & $\operatorname{sim}$ & $<3,0$ & $\operatorname{sim}$ & $\operatorname{sim}$ & $<3,0$ & $\operatorname{sim}$ & $\operatorname{sim}$ & $<10$ \\
\hline 1.4 & $8,9 \times 10^{3}$ & $\operatorname{sim}$ & 3,6 & $\operatorname{sim}$ & $\operatorname{sim}$ & $<3,0$ & $\operatorname{sim}$ & $\operatorname{sim}$ & $<10$ \\
\hline 2.0 & $4,2 \times 10^{1}$ & $\operatorname{sim}$ & $<3,0$ & $\operatorname{sim}$ & $\operatorname{sim}$ & $<3,0$ & $\operatorname{sim}$ & $\operatorname{sim}$ & $<10$ \\
\hline 2.1 & $6,72 \times 10^{3}$ & $\operatorname{sim}$ & $<3,0$ & $\operatorname{sim}$ & $\operatorname{sim}$ & $<3,0$ & $\operatorname{sim}$ & $\operatorname{sim}$ & $<10$ \\
\hline 2.2 & $2,29 \times 10^{4}$ & não & $<3,0$ & $\operatorname{sim}$ & $\operatorname{sim}$ & $<3,0$ & $\operatorname{sim}$ & $\operatorname{sim}$ & $<10$ \\
\hline 2.3 & $2,92 \times 10^{5}$ & não & 9,2 & $\operatorname{sim}$ & $\operatorname{sim}$ & $<3,0$ & $\operatorname{sim}$ & $\operatorname{sim}$ & $<10$ \\
\hline 2.4 & $7,5 \times 10^{4}$ & não & 3,6 & $\operatorname{sim}$ & $\operatorname{sim}$ & 3,6 & não & não & $<10$ \\
\hline 3.0 & $<10$ & $\operatorname{sim}$ & $<3,0$ & $\operatorname{sim}$ & $\operatorname{sim}$ & $<3,0$ & $\operatorname{sim}$ & $\operatorname{sim}$ & $<10$ \\
\hline 3.1 & $4,1 \times 10^{4}$ & não & 11 & não & $\operatorname{sim}$ & $<3,0$ & $\operatorname{sim}$ & $\operatorname{sim}$ & $<10$ \\
\hline 3.2 & $1,3 \times 10^{5}$ & não & $<3,0$ & $\operatorname{sim}$ & $\operatorname{sim}$ & $<3,0$ & $\operatorname{sim}$ & $\operatorname{sim}$ & $<10$ \\
\hline 4.0 & $<10$ & $\operatorname{sim}$ & $<3,0$ & $\operatorname{sim}$ & $\operatorname{sim}$ & $<3,0$ & $\operatorname{sim}$ & $\operatorname{sim}$ & $<10$ \\
\hline 4.1 & $7,2 \times 10^{2}$ & $\operatorname{sim}$ & $<3,0$ & $\operatorname{sim}$ & $\operatorname{sim}$ & $<3,0$ & $\operatorname{sim}$ & $\operatorname{sim}$ & $<10$ \\
\hline 4.2 & $2,57 \times 10^{4}$ & não & $<3,0$ & $\operatorname{sim}$ & $\operatorname{sim}$ & $<3,0$ & $\operatorname{sim}$ & $\operatorname{sim}$ & $<10$ \\
\hline 4.3 & $1,81 \times 10^{5}$ & não & $<3,0$ & $\operatorname{sim}$ & $\operatorname{sim}$ & $<3,0$ & $\operatorname{sim}$ & $\operatorname{sim}$ & $<10$ \\
\hline 5.0 & $<10$ & $\operatorname{sim}$ & $<3,0$ & $\operatorname{sim}$ & $\operatorname{sim}$ & $<3,0$ & $\operatorname{sim}$ & $\operatorname{sim}$ & $<10$ \\
\hline 5.1 & $1,52 \times 10^{3}$ & $\operatorname{sim}$ & 6,1 & $\operatorname{sim}$ & $\operatorname{sim}$ & 6,1 & não & não & $<10$ \\
\hline 5.2 & $1,95 \times 10^{4}$ & não & 23 & não & não & $<3,0$ & $\operatorname{sim}$ & $\operatorname{sim}$ & $<10$ \\
\hline 5.3 & $2,5 \times 10^{5}$ & não & 9,2 & $\operatorname{sim}$ & $\operatorname{sim}$ & $<3,0$ & $\operatorname{sim}$ & $\operatorname{sim}$ & $<10$ \\
\hline 5.4 & $1,23 \times 10^{5}$ & não & $<3,0$ & $\operatorname{sim}$ & $\operatorname{sim}$ & $<3,0$ & $\operatorname{sim}$ & $\operatorname{sim}$ & $<10$ \\
\hline 6.0 & $2,32 \times 10^{3}$ & $\operatorname{sim}$ & $<3,0$ & $\operatorname{sim}$ & $\operatorname{sim}$ & $<3,0$ & $\operatorname{sim}$ & $\operatorname{sim}$ & $<10$ \\
\hline 7.0 & $6,0 \times 10^{2}$ & $\operatorname{sim}$ & $<3,0$ & $\operatorname{sim}$ & $\operatorname{sim}$ & $<3,0$ & $\operatorname{sim}$ & $\operatorname{sim}$ & $<10$ \\
\hline 7.1 & $3,1 \times 10^{4}$ & não & 23 & não & não & 3,6 & não & não & $<10$ \\
\hline 7.2 & $1,78 \times 10^{5}$ & não & $<3,0$ & $\operatorname{sim}$ & $\operatorname{sim}$ & $<3,0$ & $\operatorname{sim}$ & $\operatorname{sim}$ & $<10$ \\
\hline \multirow{2}{*}{$\begin{array}{c}\text { Adequação } \\
(\%)\end{array}$} & \multirow{2}{*}{\multicolumn{2}{|c|}{$\begin{array}{c}\text { FDA (2006) } \\
46,2\end{array}$}} & & \multicolumn{2}{|c|}{ RDC 12/2001 } & & \multicolumn{2}{|c|}{ RDC 12/2001 } & \\
\hline & & & & $\begin{array}{c}<1 \text { ano } \\
88,5\end{array}$ & $\begin{array}{c}>1 \text { ano } \\
92,3\end{array}$ & & $\begin{array}{r}<1 \text { ano } \\
88,5\end{array}$ & $\begin{array}{c}>1 \text { ano } \\
88,5\end{array}$ & \\
\hline
\end{tabular}

Presença elevada de aeróbios mesófilos foi detectada em 50\% das amostras. Segundo o Compliance Program Guidance Manual da Food and Drug Administration (FDA) a contagem máxima aceitável de microrganismos aeróbios mesófilos totais em fórmulas infantis é de $10.000 \mathrm{UFC}$ por g ou $\mathrm{mL}$ de alimento [16]. Embora a análise de aeróbios mesófilos não diferencie espécies bacterianas, impossibilitando relação direta com a presença de patógenos e toxinas, um elevado número de colônias na análise em meio Plate Count Agar (PCA) indica que houve condições para o crescimento inclusive de bactérias patogênicas, já que os microrganismos patogênicos de origem alimentar são em sua maioria mesófilos [18]. Adicionalmente, a presença elevada de aeróbios mesófilos é indicativa das condições insatisfatórias de higiene na produção, com possível acúmulo de bactérias em equipamentos utilizados na reconstituição destas fórmulas [19].

Em hospitais da cidade de Salvador, na Bahia, Almeida et al. (1999) observaram o aumento de até dois ciclos logarítmicos na contagem de microrganismos aeróbios mesófilos totais quando a fórmula em pó era reconstituída [20]. No trabalho, os utensilios foram destacados como um dos principais vetores desta contaminação. Rossi et al. (2010), observaram contagem de até 4,9 × $10^{7} \mathrm{UFC} / \mathrm{g}$ ou $\mathrm{mL}$ de aeróbios mesófilos em amostras de fórmula infantil [21].

Quanto aos coliformes totais $\left(30-35^{\circ} \mathrm{C}\right)-$ grupo composto pelos gêneros Eschericbia, Enterobacter, Citrobacter e Klebsiella - a RDC no 12 apresenta níveis tolerados de contagens microbianas com valores específicos para fórmulas infantis para lactantes até 1 ano de idade e para lactantes acima de um ano [15]. Desta forma, é necessário definir se as amostras encontravam-se adequadas para o consumo nas duas situações, uma vez que as fórmulas são preparadas no lactário sem exclusividade ou separação por grupo etário, devendo-se as condições de higiene contemplar o padrão mais exigente (para lactantes menores de 1 ano de vida). Conforme os resultados, para crianças até 1 ano de idade, 11,5\% das amostras de fórmula infantil apresentaram-se inadequadas para o consumo por apresentarem presença de coliformes totais acima 
do limite indicado na RDC 12/2001 [15]. Considerando o mesmo grupo de microrganismos indicadores, duas amostras $(7,7 \%)$ apresentaram-se inadequadas para $\mathrm{o}$ consumo por crianças acima de 1 ano de idade.

No caso dos coliformes termotolerantes, também denominados coliformes a $45^{\circ} \mathrm{C}$ (contaminação mais grave, pela potencial presença de E. coli), 3 amostras $(11,5 \%)$ estavam inadequadas, segundo a legislação, para bebês de até 1 ano de idade e para crianças maiores de 1 ano. Destaca-se que tais amostras foram coletas e analisadas mais de uma vez, sendo que a coleta mínima destas foi realizada em três dias diferentes. Os resultados estiveram acima do padrão estipulado pela legislação em apenas uma das vezes em que cada uma das formulações foi obtida.

Silva et al. (2010) relatam que dados divulgados pela Food and Agriculture Organization (FAO) e a Organização Mundial da Saúde (OMS) revelaram que a presença de E. coli, coliformes totais e termotolerantes estão relacionados com uma maior probabilidade de presença de patógenos entéricos [17].

Com relação à presença de coliformes a $45^{\circ} \mathrm{C}$ (outrora denominados coliformes fecais) a RDC $\mathrm{n}^{\mathrm{o}}$ 12/2001 determina que estes microrganismos devam estar ausentes neste produto, porém as amostras 2, $5 \mathrm{e}$ 7 indicaram presença de coliformes a $45^{\circ} \mathrm{C}$ em uma das análises de cada formulação [15]. O resultado positivo em uma análise de coliformes a $45^{\circ} \mathrm{C}$ pode ser indicativo da presença de E. coli, que é considerado o exemplo mais significativo de bactéria gram negativa relacionada com diversas doenças [22]. Além disso, dados da literatura demonstram que esses microrganismos são também indicadores de falhas no processo de higienização no momento da manipulação do produto, da higienização ambiental e/ou de equipamentos. Portanto, a ausência desses microrganismos é fundamental em fórmulas lácteas, tendo em vista o estado imunológico do público-alvo [23].

Nenhuma das amostras analisadas neste trabalho apresentou contaminação por Estafilococos coagulase positiva. Dados da literatura descrevem que a bactéria Staphylococcus aureus ocorre apenas esporadicamente e em níveis muito baixos no alimento estudado [19]. Estes dados corroboram com resultado encontrado no presente estudo, no qual não foi observada a presença colônias típicas de $S$. aureus nas amostras analisadas. Mesmo com a presença de colônias não típicas (sem halos transparentes) foram realizados testes subsequentes (coagulase e catalase) não sendo confirmada a presença de Estafilococos coagulase positiva.

O estudo apontou para um total de $53,8 \%$ de amostras inadequadas ao consumo em ambas as faixas etárias, quando consideradas todas as amostras que apresentaram presença de qualquer dos microrganismos estudados acima dos limites indicados pela RDC no 12/2001 [15] e FDA [16]. Conforme os resultados apresentados, é possível inferir que, apesar de o controle higiênico sanitário estar presente em lactários no geral, existem ainda importantes falhas nestes procedimentos. Isso se deve, provavelmente, a questões como variação no grau de instrução e treinamento dos manipuladores; falhas na higienização dos equipamentos e utensílios; inadequação do controle de temperatura ambiental; inadequações no espaço de manipulação; entre outras.

Os resultados obtidos neste estudo foram divulgados para os responsáveis da unidade e feitas ações corretivas para sanar as não-conformidades encontradas em algumas das amostras. Dentre essas medidas foram realizados treinamentos com a equipe de manipuladores.

Leal [24] em pesquisa realizada no Canadá divulgou resultados importantes com relação aos manipuladores de alimentos. Foi observado que o conhecimento sobre alimento seguro era maior em manipuladores treinados. Aqueles que não recebiam treinamento apresentaram menor nível de informação, assim como os manipuladores novos na carreira (menos de um ano) ou os que trabalham há muito tempo na profissão (mais de dez anos). Estes dados reforçam a importância e o resultado positivo de um treinamento na área de segurança alimentar aos funcionários de uma unidade produtora de alimentos. Quando conduzidos de forma correta e com frequência, podem atuar na promoção, manutenção e reciclagem de conhecimentos, além de moldar as atitudes e comportamentos de manipuladores de alimentos [25].

As doenças transmitidas por alimentos servidos em hospitais são tratadas como infecção hospitalar, apresentando um grau de severidade mais elevado quando adquiridas por pacientes cujo estado de saúde já se apresenta debilitado, principalmente crianças menores de cinco anos [13]. Para esta população, portanto, o fornecimento de fórmulas infantis deve ser ainda mais controlado, visando 
oferecer um alimento o mais livre possível de contaminação microbiológica. Sabe-se que apesar do esforço da indústria ao empregar vários passos para a redução da carga microbiana no produto, as fórmulas infantis não são produtos comercialmente estéreis, e, somente quando reconstituídas em condições controladas conseguirão apresentar baixo nível de presença de microrganismos [19].

A constante investigação da presença de microrganismos indicadores e patogênicos nesse tipo de alimento é uma importante ferramenta de controle de qualidade para a garantia de um alimento seguro.

\section{CONCLUSÃO}

Dos microrganismos analisados nas 26 amostras de fórmulas infantis, 88,5\% atenderam aos parâmetros para oferecimento para lactentes menores de 1 ano de vida e 92,3\% atenderam as exigências para pacientes com mais de 1 ano de vida, segundo a RDC no 12/2001. Porém, quando observados paralelamente adequação a parâmetros desta legislação e a recomendação da FDA (2006) para aeróbios mesófilos apenas 46,2\% apresentavam contagens dos microrganismos avaliados dentro dos limites tolerados.

\section{ÓRGÃO DE FINANCIAMENTO}

Conselho Nacional de Desenvolvimento Científico e Tecnológico - CNPq. Número do Processo: 144659/2011-5.

\section{REFERÊNCIAS}

[1] Antunes AEC, Pacheco MTB. Leite para adultos: mitos e fatos frente à Ciência. São Paulo: Varela; 2009.

[2] Levy L, Bértolo H. Manual de aleitamento materno. Comitê Português para UNICEF - Comissão Nacional Iniciativa Hospitais Amigos dos Bebés [internet]. 2008 [acesso em 17 fev 2013]. Disponível em: http://www.unicef.pt/docs/manual_aleitamento.pdf

[3] Trahms CM, McKean KN. Nutrição no Estágio Inicial da Infância. In: Alimentos, Nutrição e Dietoterapia. 12.ed. Rio de Janeiro: Elsevier; 2010. p.199-211.

[4] Brasil. Agência Nacional de Vigilância Sanitária ANVISA. Resoluções RDC nos 43 e 44, de 19 de setembro de 2011. Regulamento técnico para fórmulas infantis para lactentes e Regulamento técnico para fórmulas infantis de seguimento para lactentes e crianças de primeira infância [internet]. 2011 [acesso em 15 jan 2013]. Disponível em http://portal.anvisa.gov.br

[5] Codex Alimentarius Comission. Joint FAO/WHO Food Standards Programme. Codex standard for infant formula (Codex stand 72-1981). In: Codex Alimentarius, 2.ed. Rome: FAO/WHO, 1994.

[6] Ferferbaum R, Falcão MC. Nutrição do recém-nascido. São Paulo: Atheneu; 2005.

[7] Sociedade Brasileira de Pediatria (SBP). Manual de orientação para a alimentação do lactente, pré-escolar, do escolar, do adolescente e na escola. Sociedade Brasileira de Pediatria. Departamento de Nutrologia. 2.ed. São Paulo: SBP; 2008.

[8] Silva VB, Cardoso RCV. Controle da qualidade higiênico sanitária na recepção e no armazenamento de alimentos: um estudo em escolas públicas municipais de Salvador, Bahia. Segurança Alimentar e Nutricional. 2011:18(01):43-57.

[9] Brasil. Ministério da Saúde. Secretaria de Vigilância Sanitária. Portaria no 977, de 5 de dezembro de 1998. Regulamento técnico referente as fórmulas infantis para lactentes e as fórmulas infantis de seguimento. Diário Oficial da União. 15 abr 1999; Seção1. p.90-91.

[10] Mezomo IFB. Os serviços de alimentação: planejamento e administração. 5.ed. Barueri: Manole; 2002.

[11] Euclydes MP. Nutrição do lactente: base científica para uma alimentação saudável. 3.ed. Viçosa: UFV; 2005.

[12] International Comission on Microbiological Specifications for Foods. Microorganisms in food: characteristics of microbial pathogens. London: Blackie Academic \& Professional; 1998.

[13] Rossi P. Avaliação de perigos microbiológicos no preparo de fórmulas infantis em lactário hospitalar [dissertação]. Faculdade de Engenharia de Alimentos da UNICAMP; 2007.

[14] Banwart GJ. Basic food microbiology. Westport: AVI Publishing Company; 1981. p.51-62.

[15] Brasil. Agência Nacional de Vigilância Sanitária ANVISA. Resolução RDC no12, de 02 de janeiro de 2001. Regulamento Técnico sobre padrões microbiológicos para alimentos. Diário Oficial da União. 10 jan 2001.

[16] Food and Drug Administration - FDA. U.S. Department of Health and Human Service. Compliance Program Guidance Manual. Food Composition, Standards, Labeling and Economics. Silver Spring; 2006. 
[17] Silva N, Junqueira VCA, Silveira LF, Taniw NFA. Manual de Métodos de Análise Microbiológica de Alimentos e Água. 4.ed. São Paulo: Varela; 2010.

[18] Castro, MRCC. Avaliação da qualidade microbiológica de leite humano cru recebido em Banco de Leite Humano [dissertação]. Piracicaba: Escola Superior de Agricultura "Luiz de Queiroz" da USP; 2006.

[19] Buchanan RL, Ruth O. Use of Microbiological Indicators for Assessing Hygiene Controls for the Manufacture of Powdered Infant Formula. J Food Prot. 2012;75(5):989-997.

[20] Almeida RCC, Matos CO, Almeida PF. Implementation of a HACCP system for on-site hospital preparation of infant formula. Food Control. 1999;10:181-197.

[21] Rossi P, Kabuki DY, Kuaye AY. Avaliação microbiológica do preparo de fórmula láctea infantil em lactário hospitalar. Rev. Inst. Adolfo Lutz. 2010;69(4):503509 .
[22] Nakazato G, Campos TA, Stegling EL, Brocchi M, Silveira WD.Virulence factors of avian pathogenic Escherichia coli (APEC). Pesq. Vet. Bras. 2009;7:479-486.

[23] Santos MIS, Tondo EC. Determinação de perigos e pontos críticos de controle para implantação de sistema de análise de perigos e pontos críticos de controle em lactário. Rev. Nutr. 2000;13(3):211-222.

[24] Leal D. Crescimento da alimentação fora do domicílio. Segurança Alimentar e Nutricional. 2010;17(01):123-132.

[25] Ghisleni DR, Basso C. Educação em saúde a manipuladores de duas unidades de alimentação e nutrição do município de Santa Maria/RS. Disc. Scientia. Série: Ciências da Saúde. 2008;9(1):101-108. 